\title{
The genetics of multiple sclerosis: review of current and emerging candidates
}

This article was published in the following Dove Press journal:

The Application of Clinical Genetics

7 August 2013

Number of times this article has been viewed

\author{
Maider Muñoz-Culla ${ }^{1,2}$ \\ Haritz Irizar ${ }^{1,2}$ \\ David Otaegui ${ }^{1,2}$
}

'Multiple Sclerosis Unit, Instituto Biodonostia, San Sebastián, Spain; ${ }^{2}$ Red Española de Esclerosis Múltiple (REEM), Barcelona, Spain
Correspondence: David Otaegui Instituto Biodonostia, Paseo Dr Begiristain s/n, San Sebastián 20014, Spain Tel +34943006293

Email david.otaegui@biodonostia.org
Abstract: Multiple sclerosis (MS) is a complex disease in which environmental, genetic, and epigenetic factors determine the risk of developing the disease. The human leukocyte antigen region is the strongest susceptibility locus linked to MS, but it does not explain the whole heritability of the disease. To find other non-human leukocyte antigen loci associated with the disease, high-throughput genotyping, sequencing, and gene-expression studies have been performed, producing a valuable quantity of information. An overview of the genomic and expression studies is provided in this review, as well as microRNA-expression studies, highlighting the importance of combining all the layers of information in order to elucidate the causes or pathological mechanisms occurring in the disease. Genetics in MS is a promising field that is presumably going to be very productive in the next decade understanding the cross talk between all the factors contributing to the development of MS.

Keywords: multiple sclerosis, genetics, gene expression, microRNA

\section{Introduction}

Multiple sclerosis (MS) is one of the most common causes of neurological disability in young adults, affecting more than 2.1 million people in the world. MS is a complex disease, meaning that the causes of the disease are several and are not fully understood. It is widely accepted that MS is a demyelinating disorder in which the immune system attacks the myelin, and a clear neurodegeneration component exists. Among the factors proposed to play a role in the complex interaction network that leads to MS, we can undoubtedly find genetic ones.

The first genetic factor related to the disease was the human leukocyte antigen (HLA) locus in the 1970s. This locus is located in the short arm of chromosome 6, in a region called major histocompatibility complex (MHC). The genes inside this region encode highly polymorphic cell-surface glycoproteins that are key components of the immune system. Since that first discovery, a great deal of effort has been made on understanding how this mechanism works. ${ }^{1}$ Nowadays, it is clear that HLA by itself cannot explain the whole genetic component of MS. Moreover differences by genetic $\operatorname{load}^{2}$ or $\operatorname{sex}^{3}$ have been reported, highlighting the complexity of how the HLA locus exerts its influence in the disease.

The new genomic tools arriving during the last decades confirmed the association of the HLA class II haplotype DRB1*15:01-DQA1*01:02-DQB1*06:02 with MS. More genetic factors had to be discovered, and that idea drove the research to association studies aimed at finding non-HLA genetic factors. 


\section{Association studies}

The search for non-HLA genetic factors started with a hypothesis-based candidate-gene approach. During this period, hundreds of studies were performed in these genes, some trustworthy and some more controversial.

Few conclusions about candidate genes can be ascertained from this period, but these studies were the first steps towards understanding that this kind of approach requires big collaborative projects to obtain reliable data. Moreover, these studies demonstrated the importance of taking into account the population background. ${ }^{4,5}$

Classic linkage studies were not the best approach in a disease like MS, due to the lack of big families and the fact that the expected genetic factors should have a modest effect in the phenotype in a multigenic way. With the arrival of new technologies, more ambitious projects were undertaken: the Genome-Wide Association Studies (GWAS). GWAS involved a hypothesis-free strategy that screens the whole genome by tagging linkage disequilibrium blocks. This approach use single-nucleotide polymorphism (SNP) data created from the HapMap project ${ }^{6}$ as reference to scan the whole genome and identify parts of the genome associated with the disease. It has less power than linkage studies, but better resolution, being the best approach to find differences in a multigenic disease such as MS, in which we expect that common variants are the ones contributing to the susceptibility of the disease.

Due to the number of statistical tests that will be run, these studies need huge samples to reach enough statistical power. Briefly, in these studies the $P$-value should cross a restrictive threshold (usually established at $10^{-7}$ or $10^{-8}$ ) to obtain a list of strong candidate genes that can be, finally, replicated in small populations. Some of the replications have failed, and in some cases the association has been controversial. On the other hand, pathway-based analysis has been proposed as an approach to rescue SNPs not passing the restrictive significance requirements of GWAS, ${ }^{7}$ but that might have an influence on the susceptibility combined with other SNPs. Applying this idea, Baranzini and colleagues identified for the first time several neural pathways as having a potential role in MS susceptibility by using a network-based approach in an elegant manner. ${ }^{8}$

Before August 2011, several GWAS were performed, confirming the previously known HLA DRB*1501 as having the strongest association with MS and identifying at least 14 other regions associated with the disease containing several genes. ${ }^{9-13}$ Moreover, some meta-analyses were carried out with these data, adding regions to the list of associations. ${ }^{14,15}$

However, the information coming from the different association studies is not always overlapping and/or validated. MS is a heterogeneous disease, with a wide spectrum of phenotypes. This reality affects the homogeneity of the groups created for the studies, adding noise to the data. A good and standardized clinical characterization of the patients is critical in any association genetic study. In this sense, a biomarker being able to distinguish different patient groups would help in a better classification, and so, refined data would be available. Moreover, the projects were looking for several (maybe hundreds) of tiny differences, each of them contributing in a modest way.

In August 2011, Nature published a seminal study performed by the joint efforts of the International Multiple Sclerosis Genetics Consortium and the Wellcome Trust Case Control Consortium 2. ${ }^{9}$ A total of 465,434 SNPs were studied in 9772 cases and 17376 controls, and after a refined analysis a list of non-HLA candidate genes related to MS was presented (Table 1). ${ }^{9}$ Most of the previously reported genes were confirmed and 29 new associations were described. These genes led to the conclusion that cell-mediated immune mechanisms play a primary role in the disease, and opened new ways to understand the disease based on immunological pathways and the misregulation of the immune system. From the publication of this seminal study to date, several groups have started to validate some of these results in their own populations. As a result, new candidates, such as ANKRD55, ${ }^{16}$ have emerged to join the last suggested list.

Functional studies should follow all these genetic studies in order to investigate the link between the genetic background and the pathophysiology of the disease. As an example of this, one of the genes listed in the GWAS, TNFRSF1A, has been functionally studied, with the conclusion that the MSassociated allele directs the expression of a novel form of the tumor necrosis factor (TNF)-R1 protein, which can block TNF and mimics the effect of TNF-blocking drugs. ${ }^{17}$ More functional studies that would get closer to the genetic data and clinical practice should come in the next few years to give medical relevance to the genetic discoveries.

The next revolution in the tools to study genetics came from the "-omics" techniques. Next-generation sequencing goes a step further in understanding how susceptibility is affected by candidate genes. The whole sequence of the genes will surely provide new data on how changes on DNA could affect protein function and relate them to MS. 
Table I Non-HLA candidate genes found to be associated with multiple sclerosis

\begin{tabular}{|c|c|c|c|c|}
\hline Chr & rsID & Putative gene & & k allele \\
\hline I & rs4648356 & MMELI & $\mathrm{C}$ & Previously identified \\
\hline I & rsl1810217 & EVI5 & A & Previously identified \\
\hline I & rs/335532 & CD58 & A & Previously identified \\
\hline I & rsl323292 & RGSI & $A$ & Previously identified \\
\hline I & rs7522462 & $K I F 2 I B$ & G & Previously identified \\
\hline 3 & rs2028597 & $C B L B$ & G & Previously identified \\
\hline 3 & rs2293370 & TMEM39A & G & Previously identified \\
\hline 3 & rs2243I 23 & $I L I 2 A$ & G & Previously identified \\
\hline 5 & rs6897932 & IL7R & G & Previously identified \\
\hline 5 & rs46I3763 & PTGER4 & G & Previously identified \\
\hline 6 & rs|3|9284| & OLIG3 & A & Previously identified \\
\hline 8 & rsI520333 & IL7 & G & Previously identified \\
\hline 10 & rs31II8470 & IL2RA & G & Previously identified \\
\hline 10 & rs 1250550 & ZMIZI & A & Previously identified \\
\hline II & rs650258 & CD6 & G & Previously identified \\
\hline 12 & rsl800693 & TNFRSFIA & G & Previously identified \\
\hline 12 & rs 12368653 & CYP27BI & A & Previously identified \\
\hline 12 & rs949|43 & MPHOSPH9 & G & Previously identified \\
\hline 16 & rs7200786 & CLECI 6A & A & Previously identified \\
\hline 16 & rs 13333054 & IRF8 & A & Previously identified \\
\hline 17 & rs9891119 & STAT3 & C & Previously identified \\
\hline 19 & rs8III2449 & TYK2 & G & Previously identified \\
\hline 20 & rs 2425752 & $C D 40$ & A & Previously identified \\
\hline I & rsII581062 & VCAMI & G & Novel independent \\
\hline 2 & rs 12466022 & No gene & C & Novel independent \\
\hline 2 & rs7595037 & PLEK & A & Novel independent \\
\hline 2 & rs 17174870 & MERTK & G & Novel independent \\
\hline 2 & rs 10201872 & SPI 40 & A & Novel independent \\
\hline 3 & rsIII29295a & EOMES & A & Novel independent \\
\hline 3 & rs669607 & No gene & C & Novel independent \\
\hline 3 & rs928264I & CD86 & G & Novel independent \\
\hline 5 & rs 2546890 & $I L I 2 B$ & A & Novel independent \\
\hline 6 & rs 12212193 & $\mathrm{BACH} 2$ & G & Novel independent \\
\hline 6 & rs802734 & THEMIS & A & Novel independent \\
\hline 6 & rsIII5480I & MYB & A & Novel independent \\
\hline 6 & rs 17066096 & IL22RA2 & G & Novel independent \\
\hline 6 & rs 1738074 & TAGAP & G & Novel independent \\
\hline 7 & rs354033 & ZNF746 & G & Novel independent \\
\hline 8 & rs44I087I & MYC & G & Novel independent \\
\hline 8 & rs $2019960 b$ & PVTI & G & Novel independent \\
\hline 10 & rs7923837 & HHEX & G & Novel independent \\
\hline 12 & rs 10466829 & CLECLI & A & Novel independent \\
\hline 14 & rs4902647 & ZFP36LI & G & Novel independent \\
\hline 14 & rs2300603 & BATF & A & Novel independent \\
\hline 14 & rs21I9704 & GALC & C & Novel independent \\
\hline 18 & rs7238078 & MALTI & A & Novel independent \\
\hline 19 & rs 1077667 & TNFSFI 4 & G & Novel independent \\
\hline 19 & rs874628 & MPVI TL2 & A & Novel independent \\
\hline 19 & rs2303759 & $D K K L I$ & C & Novel independent \\
\hline 20 & rs2248359 & CYP24AI & G & Novel independent \\
\hline 22 & rs2283792 & MAPKI & C & Novel independent \\
\hline 22 & rsl40522 & SCO2 & A & Novel independent \\
\hline 4 & rs2286I4 & NFKBI & G & Novel independent \\
\hline
\end{tabular}

Table I (Continued)

\begin{tabular}{lllll}
\hline Chr & rsID & $\begin{array}{l}\text { Putative gene } \\
\text { of interest }\end{array}$ & Risk allele \\
\hline II & rs630923 & CXCR5 & C & Novel independent \\
16 & rs2744I48 & SOX8 & G & Novel independent \\
17 & rs1805I5 & RPS6KBI & G & Novel independent \\
20 & rs60623I4 & TNFRSF6B & A & Novel independent \\
\hline
\end{tabular}

Adapted with permission from Macmillan Publishers Ltd: Wellcome Trust Case Control Consortium, Sawcer S, Hellenthal G, et al. Genetic risk and a primary role for cell-mediated immune mechanisms in multiple sclerosis. Nature. 201 I;476:214-219.99

Using exome sequencing, rare variants in one of the genes suggested by the 2011 GWAS, CYP27B1, have been found in MS families. ${ }^{18}$ This variant causes an arginine-to-histidine change at position $389(\mathrm{R} 389 \mathrm{H})$ of the protein and leads to complete loss of enzyme activity, resulting in lower levels of calcitriol. These results suggest that low levels of calcitriol may play a key role in the interaction of genetic and environmental factors associated with MS. However in a recent paper, Ban et al studied this association in 495 multiplex families, 2092 single affected families, 4954 cases, and 3583 controls, and were unable to find evidence supporting this association. ${ }^{19}$ Recently, using a similar approach, a family of four generations and a validation cohort of $2104 \mathrm{MS}$ trio families have been studied. The authors found a rare variant in the TYK2 gene of modest effect on MS risk affecting a subset of patients $(0.8 \%){ }^{20}$

An ambitious approach using these -omics techniques tried to elucidate the genetic and epigenetic load in MS by studying two monozygotic twins discordant for the disease. Whole-genome sequence, messenger RNA (mRNA) transcriptome, and the epigenome of $\mathrm{CD} 4^{+}$lymphocytes were studied. ${ }^{21}$ However, no new information on variants related to MS was revealed, pointing to other epigenetic characteristics, or in this concrete case to a strong environmental cause.

The immune component is undeniable in MS, and has been widely supported by experimental data (reviewed by Nylander and Hafler). ${ }^{22}$ In the last few years, several GWAS in other autoimmune diseases began to identify risk alleles, and the existence of a number of shared genes became evident. ${ }^{23}$ Taking into account the possibility of sharing susceptibility genes by chance, the statistical evidence supports the idea of the so-called "autoimmunome" network, whose genes would give a predisposition for autoimmune disease. Sophisticated bioinformatic tools, such as iCTNet (Integrative Complex Traits Network), a plugin for Cytoscape ${ }^{24}$ (one of the mostly used software packages for network visualization), have been develope ${ }^{25}$ to help researchers in the understanding of these networks. This tool allows the visualization of 
multiple relations between diseases, genes, proteins, organs, and therapeutic drugs as a network.

As an example of this immune component, an antigen called KIR4.1 has been reported recently as one of the targets of the autoimmune response in MS patients, based on evidence that antibodies against this protein have been found in serum of $46.9 \%$ of MS patients. ${ }^{26}$ However, these results have to be validated to be included in the MS paradigm, since this is the only work describing this antigen as target of the immune response in MS.

The GWAS revolution gave us exciting results, but maybe there is more to tell in this story. The Encyclopedia of DNA Elements (ENCODE) Project Consortium, ${ }^{27}$ with the aim of identifying all functional elements of the genome, has been able to assign a biochemical function to $80 \%$ of the genome. As a global conclusion from this project, we could say that what used to be called "junk DNA" due to our lack of knowledge about its function must be renamed because of the huge quantity of information these regions have about regulation and modification of the coding genes.

Regions of transcription, transcription-factor association, chromatin structure, and histone modifications have been systematically mapped, providing new information about the organization and regulation of our genes and genome. ${ }^{28}$ Interestingly, it was found that SNPs reported to be associated with disease by GWAS are enriched within noncoding functional elements. This observation suggests that GWAS data in MS should be revisited. GWAS are based on informative SNPs that are representative of regions of the genome. The candidate genes selected in the last GWAS are protein-coding genes inside the region defined by the informative SNP, but nonprotein-coding genes should also be considered as candidate genes, given that they may regulate the transcription events of the surrounding regions.

In fact, in the last decade, an increasing number of articles have described noncoding RNAs as an important piece of the gene-expression regulatory network, and their implication in several neurodegenerative and autoimmune diseases has been described (see below). In that sense, several studies highlight the importance of microRNAs (miRNAs; a noncoding family of small RNAs) in the etiology of MS, given that they regulate the expression levels of specific mRNA. Therefore, future projects combining both miRNA and gene expression might be of great interest to better understand the pathophysiology of the disease and treatment effects.

Besides noncoding RNAs, epigenetic alterations have been characterized in many diseases, including MS (reviewed by Huynh and Casaccia). ${ }^{29}$ For instance, promoter methylation status regulates the expression of several genes involved in MS pathogenesis, such as PAD2, SHP-1, and IL17A. PAD2 is overexpressed in MS patients' brain and peripheral blood due to hypomethylation of its promoter. ${ }^{30}$ Reduced $S H P-1$ expression has been found in peripheral blood mononuclear cells (PBMCs) from MS patients compared to healthy donors, and abnormally high methylation of its promoter could contribute to this deregulation. ${ }^{31}$ The expression of interleukin (IL)-17 cytokine, which has been related to MS pathophysiology, ${ }^{32}$ is regulated by promoter methylation and an intergenic enhancer. ${ }^{33}$ Moreover, an inefficient histone deacetylation has been related to an impaired remyelination in old mouse brains, ${ }^{34}$ and in MS patients increased acetylation has been detected, associated with high levels of transcriptional inhibitors of oligodendrocyte differentiation. ${ }^{35}$

In addition, an interaction of all these epigenetic mechanisms is being depicted. DNA methylation ${ }^{36}$ and histone deacetylation ${ }^{37}$ regulate the expression of miRNA, which in turn, can also regulate DNA methylation. ${ }^{38}$

To make the picture more complex, recently another genetic marker has been related to pediatric MS, the copynumber variation (CNV). In this work, ten new CNVs not overlapping with any $\mathrm{CNV}$ regions previously reported in the database of genomic variants were discovered. ${ }^{39}$ Despite not having found any causative variants, this study showed useful data suggesting that CNVs could be another character to take into account in MS.

\section{Expression studies}

Microarray analysis of gene expression has been used to explore several aspects of the pathological mechanisms involved in MS, providing the MS community with a huge quantity of data and valuable new discoveries.

Large-scale gene-expression studies performed on the brains of both MS patients and experimental autoimmune encephalomyelitis (EAE) mice have revealed genes and pathways implicated in the pathophysiology of the disease in the central nervous system (CNS).

In 1999, Whitney et al measured the expression of 5000 genes in the normal white matter and two acute lesions of brain tissue obtained in autopsy from a 46-year-old male MS patient. ${ }^{40}$ They found 62 differentially expressed genes (DEGs), 29 of them upregulated in acute lesions. These 29 were genes implicated in cell adhesion, structure and transport, myelin formation, cell growth, signaling, cell cycle, and homeostasis and immunity.

In 2001, Ibrahim et al analyzed the expression of 11,000 genes in the spinal cord of C57BL/6 EAE mice 
immunized with myelin oligodendrocyte glycoprotein 35-55. ${ }^{41}$ Mice were killed at 16 days (disease onset) and 22 days after immunization (peak of disease), and RNA was isolated from homogenized spinal cords. A total of 213 genes were found to be differentially expressed when compared to control mice, 100 of them consistently throughout the disease. The 213 genes were implicated in antigen processing and presentation, immunity, extracellular matrix, cell adhesion and matrix degradation, signal transduction, transcription, cell structure, movement, and secretion, functions in the CNS, cell division, and death.

The same year, Chabas et al confirmed in the spinal cord of EAE mice the elevated expression of osteopontin they had previously found in a large-scale sequencing experiment of cDNA libraries obtained from plaques of MS patients. ${ }^{42}$ Osteopontin-deficient mice proved to be resistant to progressive EAE.

In 2003, Graumann and colleagues performed a microarray experiment on 18 white-matter samples obtained from postmortem brains of nine MS patients and seven control cases in order to describe the earliest gene-expression changes that lead to lesion. They compared the expression of 3528 genes in normal-appearing white matter from patients and normal white matter from the corresponding brain areas of the control cases and found an upregulation of genes involved in cellular homeostasis and neural protective mechanisms triggered in ischemic preconditioning. Several HIF- $1 \alpha$ and hypoxia-induced genes, phosphatidylinositide 3-kinase/Akt pathway genes, and genes involved in preconditioning pathways were found to be overexpressed in normal-appearing white matter from patients. ${ }^{43}$ The same year, Arnett et al studied changes in gene expression related to demyelination and remyelination in mice. ${ }^{44}$ They analyzed the expression of 6000 genes in the corpus callosum of mice during diet with cuprizone (which causes demyelination), and after putting them back onto a normal diet (which allowed remyelination) in normal mice and mice lacking $T N F-\alpha$ $\left(T N F \alpha^{\prime-}\right)$. Then, they checked for gene-expression differences between demyelination and remyelination and between successful remyelination vs unsuccessful remyelination in $\mathrm{TNF}^{-/-}$mice. The most prominent alterations happened in immunological genes: MHC genes were found strongly upregulated in microglia and astrocytes during demyelination, remyelination, and $T N F-\alpha$ stimulation. MHC-null mice showed delayed remyelination and demyelination.

In 2005, Camelo et al studied the effect of the histone deacetylase inhibitory drug trichostatin (TSA) in the CNS of EAE mice. ${ }^{45}$ Trichostatin has been shown to ameliorate disability in the relapsing phase of EAE. They isolated RNA from the spinal cords of normal mice, EAE mice, and TSA-treated EAE mice, and measured the expression of 12,426 genes with microarrays. They found that TSA induced the expression of antioxidant, anti-excitotoxicity, and proneural growth and differentiation genes. In contrast, the drug inhibited target genes of the proapoptotic E2F transcription factor pathway.

Finally, in 2006 Dutta et al conducted a whole-genome gene-expression experiment in postmortem motor cortex samples from six MS patients and six healthy controls. ${ }^{46}$ They measured the expression of 33,000 genes, and 555 DEGs were identified between the nonlesion motor cortex from patients and the tissue from controls. Of the 555 genes, 488 were underexpressed and 67 overexpressed in the MS samples and Expression Analysis Systematic Explorer software classified them as being related to oxidative phosphorylation, synaptic transmission, cellular transport, the MHC, antigen presentation, antigen processing, and translational initiation. Among others, 26 mitochondrial genes and several presynaptic and postsynaptic genes of gamma-aminobutyric acidergic transmission were found to be downregulated.

Microarray studies have also been carried out on peripheral blood of MS patients with the aim of elucidating the pathogenic molecular and cellular mechanisms acting in the immune system.

In 2003, Bomprezzi et al analyzed the expression of 7500-9000 genes in PBMCs of 27 MS patients and 19 controls. ${ }^{47}$ Applying a classification algorithm, they found more than 1000 gene pairs that could distinguish MS patients from healthy controls and came up with a final list of 53 discriminatory genes. From the biological roles of the genes in the list, they concluded that the activation of autoreactive $\mathrm{T}$ cells is of primary importance in MS. The following year, Achiron et al published a microarray study of PBMCs obtained from $26 \mathrm{MS}$ patients and 18 healthy controls. ${ }^{48}$ An 1109-gene signature was identified irrespective of disease state or immunomodulatory treatment. The signature comprised genes implicated in T-cell activation and expansion, inflammation, and apoptosis. Another transcriptional signature of 721 genes distinguished MS patients in relapse from those in remission and contained genes involved in cellular recruitment, epitope spreading, and escape from regulatory immune surveillance.

In 2008, Arthur and colleagues performed a wholegenome expression experiment on whole blood of ten MS patients in relapse, another ten patients in remission, and ten healthy controls. ${ }^{49}$ They found 989 genes to be upregulated in patients in relapse. They found 1525 DEGs in patients in 
relapse when compared to healthy controls. Of these, 989 were upregulated and 536 downregulated, $A L O X 5$ and TGF $\beta 1$ showing the most significant overexpression. When comparing patients in remission with controls, 1317 genes were found to be deregulated: 655 upregulated and 662 downregulated. $A L O X 5$ was overexpressed also in remission. Among all differentially expressed genes, three had already been associated with MS: $T G F \beta 1, C D 58$, and $D B C 1$.

In summary, microarray studies on MS have provided valuable information on molecular pathways and cellular mechanisms implicated in the disease. The studies performed in the CNS of both MS patients and EAE mice have revealed deregulated genes involved in cell adhesion/structure/ movement, signaling/signal transduction, cell cycle/growth/ division/death, immunity, and antigen presentation and processing in two or more of the studies reviewed, highlighting the importance of both immune-mediated attack and a healing response of the neural/glial tissue in the pathophysiology of the disease in the CNS. On the other hand, the studies performed on peripheral blood have consistently shown a central role of T-cell activation, expansion, and inflammation, with the data coming from the DNA studies mentioned at the beginning.

However, as shown by a systematic review of geneexpression studies in MS, the reproducibility of the results between the different studies has been low; not that much at the level of molecular pathways or biological processes, but certainly for the lists of differentially expressed genes. Seven microarray studies on peripheral blood of MS patients were included in this systematic review, and from the 2017 unique genes, only 229 (11.35\%) were found to be differentially expressed in MS in the same direction in two or more studies. ${ }^{50}$

Several factors have contributed to the low reproducibility of microarray results at the differentially expressed gene level in multiple sclerosis. (1) Gene expression at the mRNA level is a very dynamic process influenced by a huge amount of variables. Moreover, interindividual variability even among healthy subjects used as reference contributes to noise. (2) Besides being a complex disease (where many genetic and environmental factors contribute to its development), MS is a very heterogeneous disease with great differences in disease progression and response to treatment between patients. The general view is that the term MS is probably an umbrella covering a whole set of different molecular pathologies converging at the clinical level. (3) Differences in starting material (PBMC vs leukocytes vs whole blood; white matter vs grey matter) and in the microarray platform used (Affymetrix, Illumina, Agilent) have contributed to low reproducibility of the results. (4) During the past decade, several algorithms have been developed for both the normalization and summarization of raw data (robust multichip algorithm, MAS5, PLIER, etc) and to obtain differentially expressed gene lists (fold-change thresholds, multiple-testing corrected $t$-test, rank product, significance analysis of microarrays, linear models for microarray data, etc). Moreover, the filtering step performed for reducing noise and relieving multiple-testing stringency has been applied in different ways in the studies. Although the most widely used filtering method implicates the elimination of low-variable genes, the removal of genes with no significant expression is also used. The problem resides in the criteria to decide whether a gene is expressed or not, which depends on the algorithm used for summarization and normalization of data. Each of the different combinations of the alternative approaches at each of the analysis steps extracts a different part of the information contained in the data, and so gives a different deregulated gene list as an output.

Two main strategies will have to be used in the future to overcome the problem of reproducibility of whole-genome expression analysis results: (1) finding criteria to create molecularly homogeneous patient groups, and (2) establishing minimum standard procedures for experimental design and data analysis. The aforementioned GWAS results could be used in the future to cluster patients based on common genetic background linked to predisposition to develop MS. Besides, more effort in the direction established by the MicroArray Quality Control (MAQC) project started by the FDA needs to be made in order to reach a consensus on the minimum set of standard procedures to follow in whole-genome expression studies (http://www.fda.gov/ScienceResearch/ BioinformaticsTools/MicroarrayQualityControlProject/ default.htm).

\section{miRNAs in MS}

One of the most widely studied types of noncoding RNAs are miRNAs. They are 20-24 nucleotide-long, single-stranded RNAs that regulate the expression of target mRNAs at posttranscriptional level. miRNAs have a role in almost all biological processes, like development, cell differentiation, proliferation, and cell death, and also in several pathological events like cancer, neurodegeneration, or autoimmunity. ${ }^{51-54}$ In the last years, several works have studied miRNA expression in MS patients in a variety of tissues (peripheral blood, brain, and CSF) and in an EAE animal model (Table 2). All these groups found alteration in miRNA-expression levels 


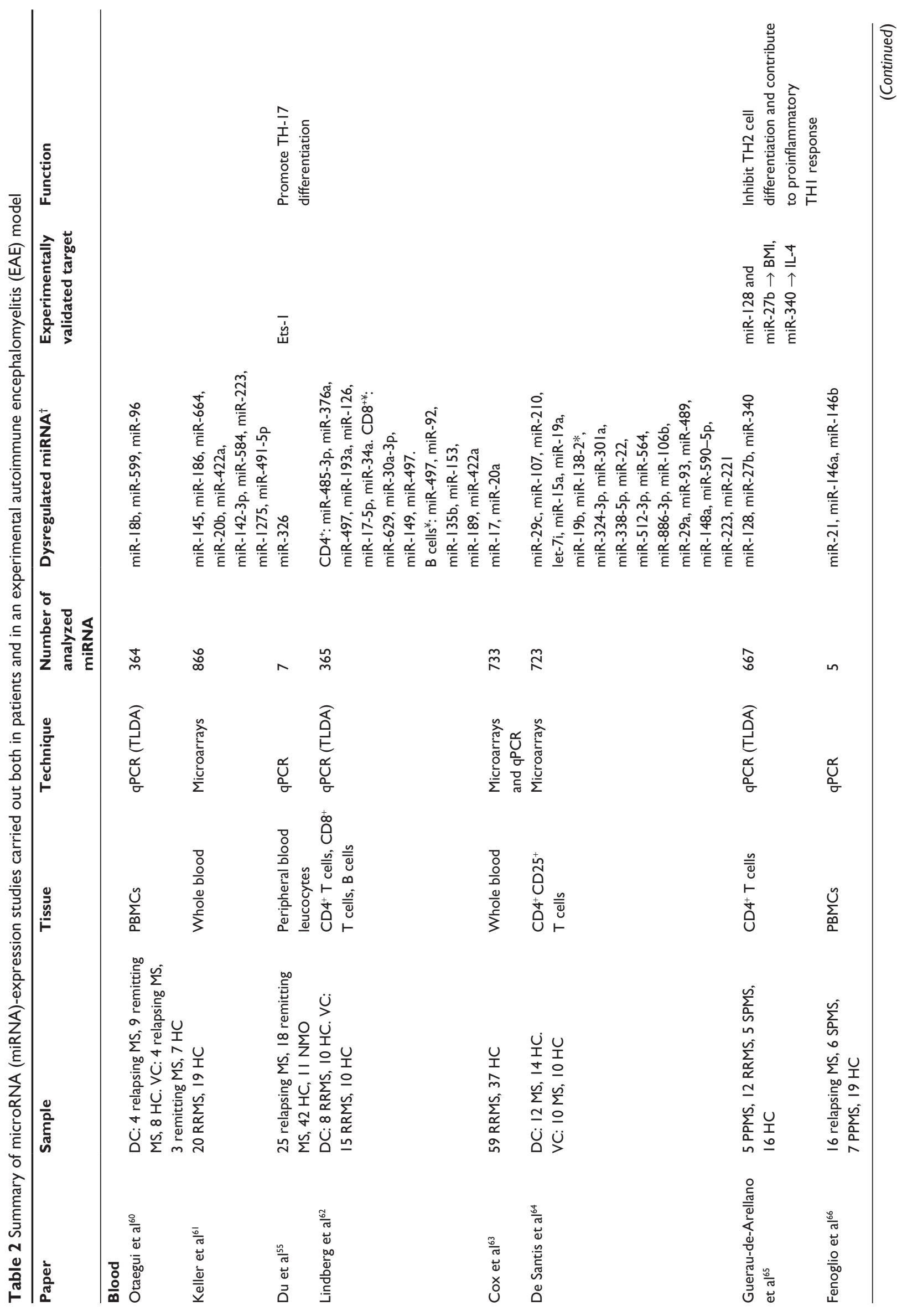




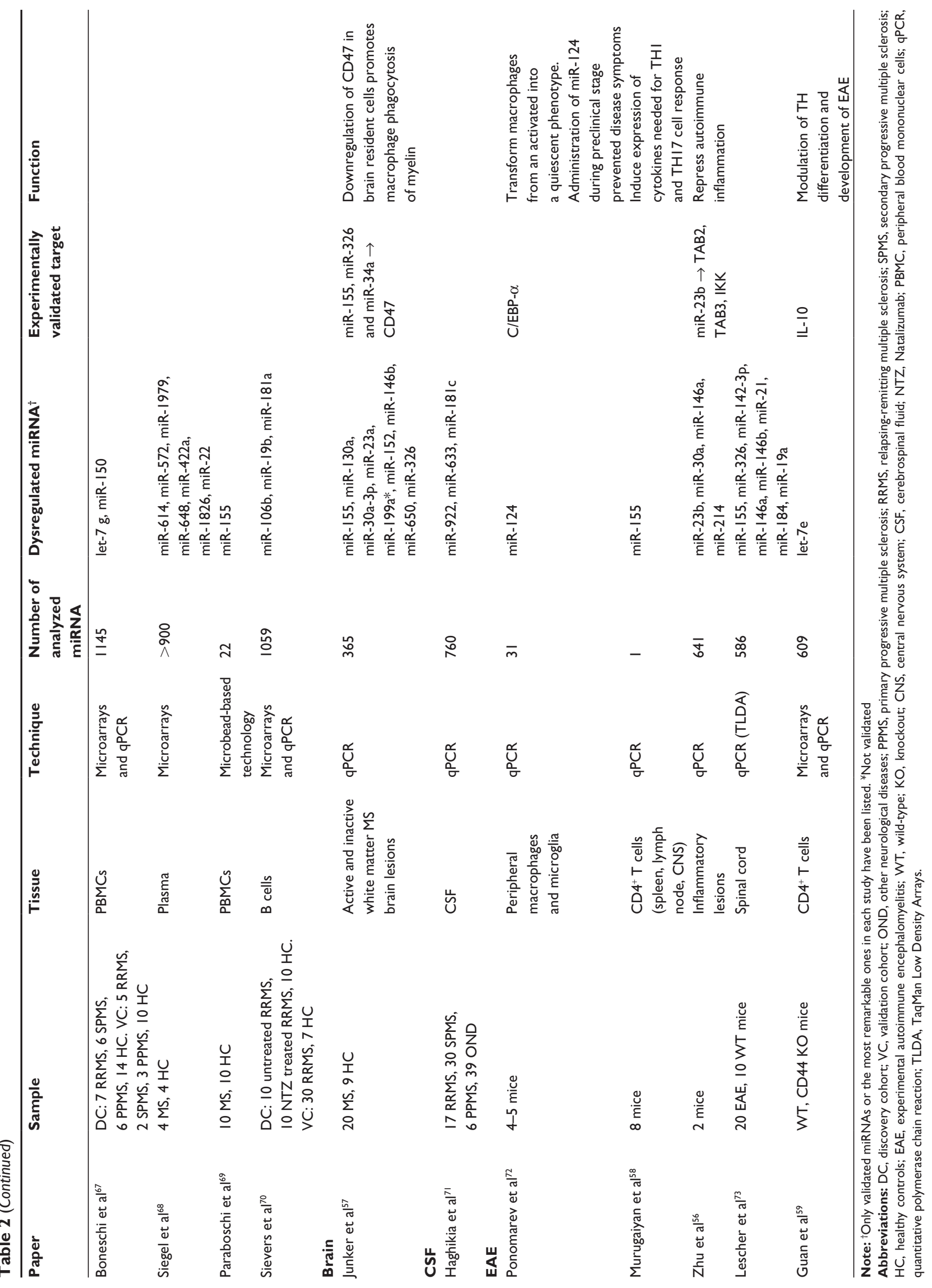


in MS patients compared to healthy controls, and therefore an implication for miRNAs seems to be evident. Yet little overlap is observed among different studies, probably due to the difference in the miRNA-profiling technology, the complexity of the tissue being studied, and the relatively small sample size of all studies (Table 2).

Several works have been able to establish a concrete function for some miRNAs related to MS. For instance, $\mathrm{Du}$ and colleagues found that miR-326 was upregulated specifically in MS patients with relapse (not in remitting patients) and more prominently in $\mathrm{CD}^{+} \mathrm{T}$ cells compared to $\mathrm{CD} 8^{+} \mathrm{T}$ cells and non-T cells. ${ }^{55}$ Then, they demonstrated that miR-326 expression in $\mathrm{CD}^{+} \mathrm{T}$ cells promotes T-helper (TH)-17 differentiation by targeting Ets-1, a known negative regulator of TH-17 cells. EAE pathology was milder in mice receiving an miR-326 inhibitor, and in fact downregulation of this miRNA was observed in a group of patients having received glucocorticoid treatment for the relapse. As the authors discuss, all these observations suggest that miR-326 is involved in the acute phase of the disease, and furthermore is under treatment effect. Another study also described a link between IL-17-associated inflammation with miRNA regulation. Researchers observed a downregulation of miR-23b in several autoimmune diseases or their animal models (EAE included) due to high expression of IL-17.56

Another noteworthy study analyzed miRNA-expression profiles in active and inactive MS lesions. Using luciferase assays, the researchers found that miR-155, miR-326, and miR-34a targeted CD47 3'UTR. ${ }^{57}$ The authors proposed that the overexpression of these three miRNAs in MS brains promotes the downregulation of CD47 on brain resident cells, thereby triggering macrophage phagocytosis of the myelin. miRNAs are also involved in the differentiation of $\mathrm{CD}^{+} \mathrm{T}$ cells, as reported by studies in animal models. These demonstrated that miR-155 and let-7e shift $\mathrm{CD}^{+} \mathrm{T}$ cell polarization towards a TH-1 and TH-17 phenotype (Table 2). ${ }^{58,59}$

All these works show how miRNAs are involved in the misregulated immune system in MS and may help us to better understand the mechanisms of the pathology. At the same time, they offer a new research field to find another piece of the MS pathophysiology puzzle.

\section{Concluding remarks}

To cut a long story short, the genetic history in MS during the last 40 years shed light on the etiology of the disease, highlighting the importance of the collaborative projects, and without any doubt, bringing out the complexity of MS. In the future, we will talk about MS gene panels for resequencing containing around 100-150 genes, but with this information we will be covering only part of the genetic component. The study of epigenetic factors such as methylation, CNV, and posttranscriptional modifications, which in some way are the hinge that links genetic background and environment or lifestyle, are some of the challenges for the next decade. Furthermore, validation studies, meta-analysis and even additional GWAS are being performed, and thus in coming years we might see the discovery of new candidate genes not only related to the risk but also to the evolution of the disease and to the drug response.

On the other hand, learning from other complex diseases like Parkinson's, we might see in the future a scenario where small groups of MS patients have a clear genetic component that explains the disease. Next-generation sequencing techniques and family studies will probably clarify this picture. The past has taught us that these challenges should be tackled by joint efforts, and that in this way the next decade will possibly come with promising and exciting results in the field of MS genetics.

\section{Disclosure}

The authors report no conflicts of interest in this work.

\section{References}

1. McElroy JP, Oksenberg JR. Multiple sclerosis genetics 2010. Neurol Clin. 2011;29:219-231.

2. Barcellos LF, Oksenberg JR, Begovich AB, et al. HLA-DR2 dose effect on susceptibility to multiple sclerosis and influence on disease course. Am J Hum Genet. 2003;72:710-716.

3. Irizar H, Muñoz-Culla M, Zuriarrain O, et al. HLA-DRB1*15:01 and multiple sclerosis: a female association? Mult Scler. 2012;18: 569-577.

4. Otaegui D, Sáenz A, Camaño P, et al. CD24 V/V is an allele associated with the risk of developing multiple sclerosis in the Spanish population. Mult Scler. 2006;12:511-514.

5. Fozza C, Zoledzieska M, Pitzalis M, et al. TCRBV20S1 polymorphism does not influence the susceptibility to type 1 diabetes and multiple sclerosis in the Sardinian population. Immunogenetics. 2012;64: $153-154$.

6. Frazer KA, Ballinger DG, Cox DR, et al. A second generation human haplotype map of over 3.1 million SNPs. Nature. 2007;449:851-861.

7. Wang K, Li M, Bucan M. Pathway-based approaches for analysis of genomewide association studies. Am J Hum Genet. 2007;81: 1278-1283.

8. Baranzini SE, Wang J, Gibson RA, et al. Genome-wide association analysis of susceptibility and clinical phenotype in multiple sclerosis. Hum Mol Genet. 2009;18:767-778.

9. Wellcome Trust Case Control Consortium, Sawcer S, Hellenthal G, et al Genetic risk and a primary role for cell-mediated immune mechanisms in multiple sclerosis. Nature. 2011;476:214-219.

10. International Multiple Sclerosis Genetics Consortium, Hafler DA, Compston A, et al. Risk alleles for multiple sclerosis identified by a genomewide study. $N$ Engl J Med. 2007;357:851-862. 
11. The Australia and New Zealand Multiple Sclerosis Genetics Consortium (ANZgene). Genome-wide association study identifies new multiple sclerosis susceptibility loci on chromosomes 12 and 20. Nat Genet. 2009;41:824-828.

12. Jakkula E, Leppä V, Sulonen AM, et al. Genome-wide association study in a high-risk isolate for multiple sclerosis reveals associated variants in STAT3 gene. Am J Hum Genet. 2010;86:285-291.

13. Matesanz F, Gonzalez-Perez A, Lucas M, et al. Genome-wide association study of multiple sclerosis confirms a novel locus at $5 \mathrm{p} 13.1$. PLoS One. 2012;7:e36140.

14. De Jager PL, Jia X, Wang J, et al. Meta-analysis of genome scans and replication identify CD6, IRF8 and TNFRSF1A as new multiple sclerosis susceptibility loci. Nat Genet. 2009;41:776-782.

15. Patsopoulos NA, Esposito F, Reischl J, et al. Genome-wide meta-analysis identifies novel multiple sclerosis susceptibility loci. Ann Neurol. 2011;70:897-912.

16. Lill CM, Schjeide BM, Graetz C, et al. Genome-wide significant association of ANKRD55 rs6859219 and multiple sclerosis risk. J Med Genet. 2013;50:140-143.

17. Gregory AP, Dendrou CA, Attfield KE, et al. TNF receptor 1 genetic risk mirrors outcome of anti-TNF therapy in multiple sclerosis. Nature. 2012;488:508-511.

18. Ramagopalan SV, Dyment DA, Cader MZ, et al. Rare variants in the CYP27B1 gene are associated with multiple sclerosis. Ann Neurol. 2011;70:881-886.

19. Ban M, Caillier S, Mero IL, et al. No evidence of association between mutant alleles of the CYP27B1 gene and multiple sclerosis. Ann Neurol. Epub December 13, 2012.

20. Dyment DA, Cader MZ, Chao MJ, et al. Exome sequencing identifies a novel multiple sclerosis susceptibility variant in the TYK2 gene. Neurology. 2012;79:406-411.

21. Baranzini SE, Mudge J, van Velkinburgh JC, et al. Genome, epigenome and RNA sequences of monozygotic twins discordant for multiple sclerosis. Nature. 2010;464:1351-1356

22. Nylander A, Hafler DA. Multiple sclerosis. J Clin Invest. 2012;122: 1180-1188.

23. Baranzini SE. The genetics of autoimmune diseases: a networked perspective. Curr Opin Immunol. 2009;21:596-605.

24. Smoot ME, Ono K, Ruscheinski J, Wang PL, Ideker T. Cytoscape 2.8: new features for data integration and network visualization. Bioinformatics. 2011;27:431-432.

25. Wang L, Khankhanian P, Baranzini SE, Mousavi P. iCTNet: a Cytoscape plugin to produce and analyze integrative complex traits networks. $B M C$ Bioinformatics. 2011;12:380.

26. Srivastava R, Aslam M, Kalluri SR, et al. Potassium channel KIR4.1 as an immune target in multiple sclerosis. $N$ Engl J Med. 2012;367: $115-123$.

27. ENCODE Project Consortium. The ENCODE (Encyclopedia of DNA Elements) Project. Science. 2004;306:636-640.

28. Dunham I, Kundaje A, Aldred SF, et al. An integrated encyclopedia of DNA elements in the human genome. Nature. 2012;489:57-74.

29. Huynh JL, Casaccia P. Epigenetic mechanisms in multiple sclerosis: implications for pathogenesis and treatment. Lancet Neurol. 2013;12: 195-206.

30. Calabrese R, Zampieri M, Mechelli R, et al. Methylation-dependent PAD2 upregulation in multiple sclerosis peripheral blood. Mult Scler. 2012;18:299-304.

31. Kumagai C, Kalman B, Middleton FA, Vyshkina T, Massa PT. Increased promoter methylation of the immune regulatory gene SHP-1 in leukocytes of multiple sclerosis subjects. J Neuroimmunol. 2012;246: 51-57.

32. Iwakura $Y$, Ishigame $H$, Saijo $S$, Nakae S. Functional specialization of interleukin-17 family members. Immunity. 2011;34:149-162.

33. Thomas RM, Sai H, Wells AD. Conserved intergenic elements and DNA methylation cooperate to regulate transcription at the il17 locus. J Biol Chem. 2012;287:25049-25059.
34. Shen S, Sandoval J, Swiss VA, et al. Age-dependent epigenetic control of differentiation inhibitors is critical for remyelination efficiency. Nat Neurosci. 2008;11:1024-1034.

35. Pedre X, Mastronardi F, Bruck W, Lopez-Rodas G, Kuhlmann T, Casaccia P. Changed histone acetylation patterns in normal-appearing white matter and early multiple sclerosis lesions. J Neurosci. 2011;31: 3435-3445.

36. Han L, Witmer PD, Casey E, Valle D, Sukumar S. DNA methylation regulates microRNA expression. Cancer Biol Ther. 2007;6:1284-1288.

37. Scott GK, Mattie MD, Berger CE, Benz SC, Benz CC. Rapid alteration of microRNA levels by histone deacetylase inhibition. Cancer Res. 2006;66:1277-1281.

38. Qin H, Zhu X, Liang J, et al. MicroRNA-29b contributes to DNA hypomethylation of CD4+ T cells in systemic lupus erythematosus by indirectly targeting DNA methyltransferase 1. J Dermatol Sci. 2013;69: 61-67.

39. McElroy J, Krupp L, Johnson B, et al. Copy number variation in pediatric multiple sclerosis. Mult Scler. Epub December 13, 2012.

40. Whitney LW, Becker KG, Tresser NJ, et al. Analysis of gene expression in mutiple sclerosis lesions using cDNA microarrays. Ann Neurol. 1999;46:425-428.

41. Ibrahim SM, Mix E, Bottcher T, et al. Gene expression profiling of the nervous system in murine experimental autoimmune encephalomyelitis. Brain. 2001;124:1927-1938.

42. Chabas D, Baranzini SE, Mitchell D, et al. The influence of the proinflammatory cytokine, osteopontin, on autoimmune demyelinating disease. Science. 2001;294:1731-1735.

43. Graumann U, Reynolds R, Steck AJ, Schaeren-Wiemers N. Molecular changes in normal appearing white matter in multiple sclerosis are characteristic of neuroprotective mechanisms against hypoxic insult. Brain Pathol. 2003;13:554-573.

44. Arnett HA, Wang Y, Matsushima GK, Suzuki K, Ting JP. Functional genomic analysis of remyelination reveals importance of inflammation in oligodendrocyte regeneration. J Neurosci. 2003;23:9824-9832.

45. Camelo S, Iglesias AH, Hwang D, et al. Transcriptional therapy with the histone deacetylase inhibitor trichostatin A ameliorates experimental autoimmune encephalomyelitis. J Neuroimmunol. 2005;164:10-21.

46. Dutta R, McDonough J, Yin X, et al. Mitochondrial dysfunction as a cause of axonal degeneration in multiple sclerosis patients. Ann Neurol. 2006;59:478-489.

47. Bomprezzi R, Ringner M, Kim S, et al. Gene expression profile in multiple sclerosis patients and healthy controls: identifying pathways relevant to disease. Hum Mol Genet. 2003;12:2191-2199.

48. Achiron A, Gurevich M, Friedman N, Kaminski N, Mandel M. Blood transcriptional signatures of multiple sclerosis: unique gene expression of disease activity. Ann Neurol. 2004;55:410-417.

49. Arthur AT, Armati PJ, Bye C, et al. Genes implicated in multiple sclerosis pathogenesis from consilience of genotyping and expression profiles in relapse and remission. BMC Med Genet. 2008;9:17.

50. Kemppinen AK, Kaprio J, Palotie A, Saarela J. Systematic review of genome-wide expression studies in multiple sclerosis. BMJ Open. 2011;1:e000053.

51. Huntzinger E, Izaurralde E. Gene silencing by microRNAs: contributions of translational repression and mRNA decay. Nat Rev Genet. 2011;12:99-110.

52. Salta E, De SB. Non-coding RNAs with essential roles in neurodegenerative disorders. Lancet Neurol. 2012;11:189-200.

53. O’Connell RM, Rao DS, Chaudhuri AA, Baltimore D. Physiological and pathological roles for microRNAs in the immune system. Nat Rev Immunol. 2010;10:111-122.

54. Ryan BM, Robles AI, Harris CC. Genetic variation in microRNA networks: the implications for cancer research. Nat Rev Cancer. 2010;10: 389-402.

55. Du C, Liu C, Kang J, et al. MicroRNA miR-326 regulates T(H)-17 differentiation and is associated with the pathogenesis of multiple sclerosis. Nat Immunol. 2009;10:1252-1259. 
56. Zhu S, Pan W, Song X, et al. The microRNA miR-23b suppresses IL17-associated autoimmune inflammation by targeting TAB2, TAB3 and IKK-alpha. Nat Med. 2012;18:1077-1086.

57. Junker A, Krumbholz M, Eisele S, et al. MicroRNA profiling of multiple sclerosis lesions identifies modulators of the regulatory protein CD47. Brain. 2009; 132:3342-3352.

58. Murugaiyan G, Beynon V, Mittal A, Joller N, Weiner HL. Silencing microRNA-155 ameliorates experimental autoimmune encephalomyelitis. J Immunol. 2011;187:2213-2221.

59. Guan H, Fan D, Mrelashvili D, et al. MicroRNA let-7e is associated with the pathogenesis of experimental autoimmune encephalomyelitis. Eur J Immunol. 2013;43:104-114.

60. Otaegui D, Baranzini SE, Armañanzas R, et al. Differential micro RNA expression in PBMC from multiple sclerosis patients. PLoS One. 2009;4:e6309.

61. Keller A, Leidinger P, Lange J, et al. Multiple sclerosis: microRNA expression profiles accurately differentiate patients with relapsingremitting disease from healthy controls. PLoS One. 2009;4:e7440.

62. Lindberg RL, Hoffmann F, Mehling M, Kuhle J, Kappos L. Altered expression of miR-17-15p in CD4+ lymphocytes of relapsing-remitting multiple sclerosis patients. Eur J Immunol. 2010;40:888-898.

63. Cox MB, Cairns MJ, Gandhi KS, et al. MicroRNAs miR-17 and miR-20a inhibit T cell activation genes and are under-expressed in MS whole blood. PLoS One. 2010;5:e12132.

64. De Santis G, Ferracin M, Biondani A, et al. Altered miRNA expression in T regulatory cells in course of multiple sclerosis. $J$ Neuroimmunol. 2010;226:165-171.

65. Guerau-de-Arellano M, Smith KM, Godlewski J, et al. Micro-RNA dysregulation in multiple sclerosis favours pro-inflammatory T-cellmediated autoimmunity. Brain. 2011;134:3578-3589.
66. Fenoglio C, Cantoni C, De RM, et al. Expression and genetic analysis of miRNAs involved in CD4+ cell activation in patients with multiple sclerosis. Neurosci Lett. 2011;504:9-12.

67. Martinelli-Boneschi F, Fenoglio C, Brambilla P, et al. MicroRNA and mRNA expression profile screening in multiple sclerosis patients to unravel novel pathogenic steps and identify potential biomarkers. Neurosci Lett. 2012;508:4-8.

68. Siegel SR, Mackenzie J, Chaplin G, Jablonski NG, Griffiths L. Circulating microRNAs involved in multiple sclerosis. Mol Biol Rep. 2012;39: 6219-6225.

69. Paraboschi EM, Solda G, Gemmati D, et al. Genetic association and altered gene expression of mir-155 in multiple sclerosis patients. Int $J$ Mol Sci. 2011;12:8695-8712.

70. Sievers C, Meira M, Hoffmann F, Fontoura P, Kappos L, Lindberg RL. Altered microRNA expression in B lymphocytes in multiple sclerosis: towards a better understanding of treatment effects. Clin Immunol. 2012;144:70-79

71. Haghikia A, Haghikia A, Hellwig K, et al. Regulated microRNAs in the CSF of patients with multiple sclerosis: a case-control study. Neurology. 2012;79:2166-2170.

72. Ponomarev ED, Veremeyko T, Barteneva N, Krichevsky AM, Weiner HL. MicroRNA-124 promotes microglia quiescence and suppresses EAE by deactivating macrophages via the C/EBP-alpha-PU.1 pathway. Nat Med. 2011;17:64-70.

73. Lescher J, Paap F, Schultz V, et al. MicroRNA regulation in experimental autoimmune encephalomyelitis in mice and marmosets resembles regulation in human multiple sclerosis lesions. J Neuroimmunol. 2012;246 27-33.
The Application of Clinical Genetics

\section{Publish your work in this journal}

The Application of Clinical Genetics is an international, peer-reviewed open access journal that welcomes laboratory and clinical findings in the field of human genetics. Specific topics include: Population genetics; Functional genetics; Natural history of genetic disease; Management of genetic disease; Mechanisms of genetic disease; Counseling and ethical

\section{Dovepress}

issues; Animal models; Pharmacogenetics; Prenatal diagnosis; Dysmorphology. The manuscript management system is completely online and includes a very quick and fair peer-review system, which is all easy to use. Visit http://www.dovepress.com/testimonials.php to read real quotes from published authors 